\title{
Notch3 inhibition enhances sorafenib cytotoxic efficacy by promoting GSK3 $\beta$ phosphorylation and p21 down-regulation in hepatocellular carcinoma
}

\author{
Catia Giovannini ${ }^{1,2}$, Michele Baglioni ${ }^{1,2}$, Marco Baron Toaldo ${ }^{3}$, Cristiano Ventrucci $^{1}$, \\ Stefania D'Adamo ${ }^{1}$, Mario Cipone ${ }^{3}$, Pasquale Chieco ${ }^{1}$, Laura Gramantieri ${ }^{1, *}$ and \\ Luigi Bolondi, ${ }^{1, *}$. \\ ${ }^{1}$ Center for Applied Biomedical Research (CRBA), S.Orsola-Malpighi University Hospital, Bologna, Italy; \\ 2 Department of Clinical Medicine University of Bologna, Bologna, Italy; \\ ${ }^{3}$ Department of Veterinary Medical Science University of Bologna, Bologna, Italy. \\ * Contributed equally to this work. \\ Correspondence to: C Giovannini, email: catia.giovannini4@unibo.it
}

Keywords: ERK, drug resistance, angiogenesis, cell proliferation, Notch3

Received: July 30, $2013 \quad$ Accepted: August 22, $2013 \quad$ Published: August 24, 2013

This is an open-access article distributed under the terms of the Creative Commons Attribution License, which permits unrestricted use, distribution, and reproduction in any medium, provided the original author and source are credited.

\section{ABSTRACT:}

Sorafenib (Nexavar), a multiple kinase inhibitor, is the only clinically approved drug for patients with advanced HCC. However, its therapeutic success is limited by the emergence of drug resistance. Here we found that p21 and pGSK3 $\beta^{\text {serg }}$ are major players in the resistance to sorafenib. We recently reported that aberrant Notch3 expression in HCC contributes to doxorubicin resistance in vitro and, therefore, we focused on the mechanisms that associate Notch 3 to acquired drug resistance. In this study we first found that Notch 3 inhibition significantly increased the apoptosis inducing effect of sorafenib in HCC cells via specific down-regulation of p21 and up-regulation of pGSK3 $\beta^{\text {ser. }}$. Using a mouse xenograft model we further found that Notch 3 depletion combined with 21 days of sorafenib treatment exerts a substantial antitumor effect in vivo. Interestingly, we showed that, upon exposure to sorafenib treatment, Notch 3 depleted xenografts maintain lower levels of p21 and higher levels of pGSK3 $\beta^{\text {serg }}$ than control xenografts. Thus, this study demonstrated that inhibition of Notch 3 signaling prevents HCC-mediate drug resistance and sensitizes HCC cells to sorafenib. Finally, we validated our in vitro and in vivo results in primary human HCCs showing that Notch 3 protein expression positively correlated with p21 protein expression and negatively correlated with pGSK3 $\beta^{\text {ser9 }}$ expression. In conclusion, the results presented in this study demonstrated that Notch 3 silencing enhances the effect of sorafenib by overcoming drug resistance. Notch3 inhibition in combination with sorafenib can be a promising strategy for treatment of HCC.

\section{INTRODUCTION}

Hepatocellular carcinoma (HCC) is a highly malignant liver tumor that most often arises in cirrhotic livers and is quite unrensponsive to common chemotherapeutic agents. In liver cirrhosis, which is today considered a premalignant condition, it is often possible to observe early signs of derangement in the same proliferative and apoptotic pathways driving the growth of HCC cells. Therefore, chemotherapeutic strategies cannot be selective for neoplastic cells and, given the central importance of this organ, liver failure is a deadly complication of HCC therapy. The recent introduction of molecularly targeted drugs has opened a new scenario for HCC treatment. In particular, sorafenib, a multi-kinase inhibitor mainly acting on vascular cells, was shown to significantly improve survival in patients with advanced HCC (SHARP and ASAP), even though prognosis still remained dismal with mean survival rates assessing at 10 and 6.2 months in the different settings (contr 
sharp and asap) $[1,2]$. Thus the exploration of possible combinations between targeted drugs whose effects are addictive or synergic, raises the possibility to find effective combinations with low toxicity. Accordingly, molecules expressed in HCC tissue and not in cirrhosis represent targets of considerable interest to preserve non neoplastic tissue and reduce side effects. The Notch 3 receptor is aberrantly expressed in nearly $80 \%$ of HCCs with a negligible expression in normal liver as well as in cirrhotic tissue surrounding HCC [3]. Given the fundamental roles played by Notch in cell fate during embryonic development, it is not a surprise that alterations in Notch signaling have been associated with tumor development [4]. Inhibition of Notch expression by antisense retrovirus or pharmacologic blocking of $\gamma$-secretase activity has shown striking antineoplastic effects in Notch-expressing transformed cells in vitro and in xenograft models [5, 6]. Recently, we showed that Notch3 depleted HCC cells have the same rate of apopotosis of control cells. However, Notch3 silencing in liver cancer cells was able to strongly enhance the therapeutic effects of doxorubicin by up-regulating p53-dependent apoptosis [7]. With regard to HCC resistance to chemotherapeutic agents, we hypothesized that Notch3 may function as a positive factor for multi-drug resistance. Indeed there is evidence that Notch3 over-expression confers resistance to carboplatin and is related to the recurrence of ovarian cancer [8].

The main purposes of the present study are to assess whether Notch3 inhibition enhances sorafenib effects in $\mathrm{HCC}$ and to individuate which molecular pathways are interacting in their therapeutic effects.

We showed that the specific block of Notch3 signaling with shRNA enhanced sensitivity to sorafenib in vitro and in vivo. These data suggest that Notch 3 inhibition holds promise as an additional strategy to improve molecular cancer therapy, in particular when resistance to and/or escape from existing therapies evolve.

\section{RESULTS}

\section{Notch3 KD enhances apoptosis of Sorafenib treated $\mathrm{HCC}$ cells.}

Sorafenib was approved for HCC therapy in 2007 and represents the standard of care for patients with advanced-stage disease [9]. Sorafenib slows tumor growth by blocking different signaling pathways involved in cell proliferation [10] and angiogenesis.

We initially explored the effects of Notch3 ablation (Fig. 1A) on sorafenib activity in the two HCC cell lines used in this study. Trypan dye uptake in multiple experiments revealed that after $72 \mathrm{~h}$ of sorafenib exposure the mortality due to Notch $3 \mathrm{KD}$ increased by 3,8 and 5 fold in HepG2 and Huh7 cells respectively (Fig.1B). We found that sorafenib treatment in Notch3 KD cells resulted in an increase of Caspase-8, Caspase-9 and Caspase-3 cleavage in conjunction with enhance Annexin V-FITC intensity (Fig.1C and 1E) but LDH was not increased in the medium when compared to GL2 cells (Suppl. Fig 1C). Taken together these results indicate that sorafenib treated Notch3 KD cells die because of apoptosis. Next we better examined the contributions of either apoptotic pathways for sorafenib induced apoptosis using Caspase-8 and Caspase-9 selective inhibitors (Fig. 1D). Annexin V-FITC staining showed a reduction in apoptosis in cells treated with caspase 8 inhibitor both in GL2 and in Notch3 depleted cells whereas caspase 9 inhibitor affects apoptosis only in Notch3 silenced cells (Fig. 1E) in line with the high levels of p53 (Fig. 1A) [7]. Taken together our data show that in Notch3 depleted cells sorafenib activates both intrinsic and extrinsic apoptotic pathway.

\section{Effects of Notch3 depletion and sorafenib treatment on in vivo tumor growth and angiogenesis.}

Targeting tumor vasculature as a cancer therapy is an established concept to benefit patients with a wide variety of tumor types because tumor angiogenesis implies a rapidly growing tumor.

In addition to targeting Raf serine /threonine kinases sorafenib inhibits several RTKs known to promote angiogenesis, as VEGFR2, in human endothelial cells [11]. Interestingly, both VEGFR2 and CD31, a transmembrane molecule expressed on endothelial cells, have been shown to transduce signals that mediate angiogenesis, vascular remodelling and cellular proliferation and their expression correlates with prognosis in HCC [12]. Our experiments showed that the treatment with sorafenib $(60 \mathrm{mg} / \mathrm{kg} / \mathrm{d})$ for 21 days inhibited tumour growth significantly more in both Huh7 $(p=0.04)$ and HepG2 $(p=0.01)$ Notch3 depleted xenografts than in GL2 xenografts. (Fig.2A). Indeed, after 21 days of sorafenib exposure Notch3 depleted xenografts showed a much lower proliferation and vessel density than Notch3 undepleted ones, as shown by immuno-staining for Ki67, VEGFR2 and CD31 (Fig.2B-E).

\section{Notch3 and Sorafenib share common pathways}

To better investigate the mechanisms associated with the enhanced sorafenib sensitivity observed in Notch3 depleted cells, we first examined the phosphorylation status of well established targets of sorafenib such as ERK1/2 and Akt [13] in Notch3 KD cells. As shown in Figures 3A \& 3B, GL2 and Notch3 KD cells had very similar expression of p-ERK1/2 and p-Akt in either untreated or sorafenib treated cells, suggesting that these molecules are not associated with the response to sorafenib in Notch3 KD cells. We next examined GSK3 $\beta$, a kinase 
activated by sorafenib, and its inhibitory phosphorylation status ( $\mathrm{pGSK} 3 \beta^{\mathrm{Ser} 9}$ ). We found that $\mathrm{pGSK} 3 \beta^{\mathrm{Ser} 9}$ was markedly increased in Notch3 KD cells compared with
GL2 cells (Fig.3A) and that the high pGSK3 $\beta^{\text {Ser9 }}$ levels were maintained in response to sorafenib exposure (Fig.3B).

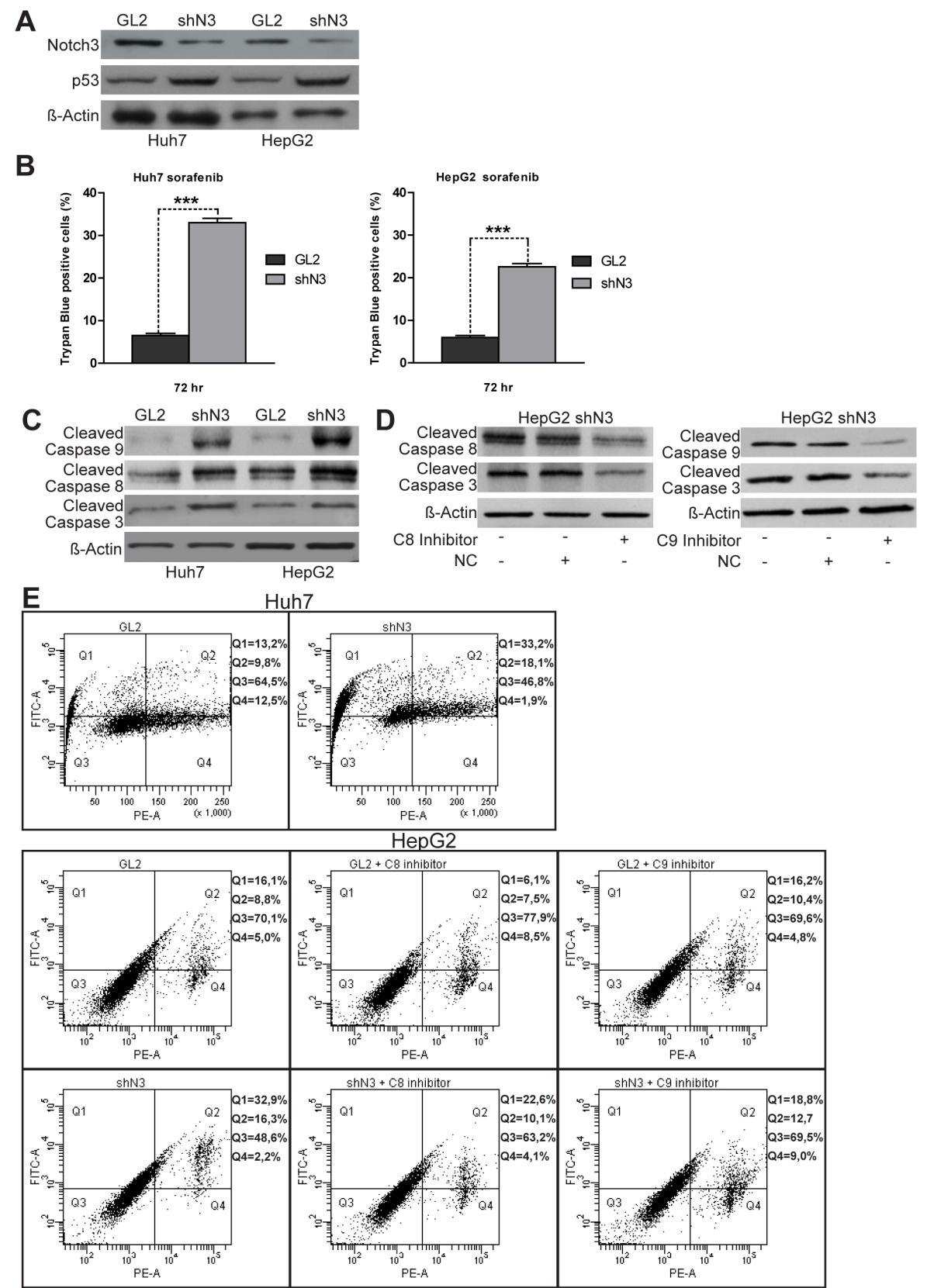

Figure 1: Notch3 KD enhances the proapoptotic effect of sorafenib in vitro. (A) Efficacy of Notch3 KD and p53 expression was measured by western blotting in Huh7 and HepG2 cells. (B) The viabilities of control and Notch3 KD Huh7 and HepG2 cells in response to $4 \mu \mathrm{M}$ of sorafenib were quantified by trypan blue uptake after $72 \mathrm{~h}$ of treatment. Results are the mean of three independent experiments (+/- S.E.). P values (by two tailed student's t test) were $<0.001$ for shN3 vs GL2 in both the analyzed cell lines. (C) shN3 and GL2 control cells were treated with $4 \mu \mathrm{M}$ of Sorafenib for $72 \mathrm{~h}$ and the levels of Cleaved Caspase 8, 9, 3 and Actin were analyzed by western blotting. (D) Expression levels of Cleaved Caspase 8,9 and 3 were evaluated in HepG2 Notch3 silenced cells treated with $4 \mu \mathrm{M}$ of sorafenib for 72 $\mathrm{h}$ and $10 \mu \mathrm{M}$ of caspase inhibitors or negative control to test inhibitors efficacy. (E) After treatment with $4 \mu \mathrm{M}$ of sorafenib for $72 \mathrm{~h}$ HepG2 and Huh7 cells were labeled with annexin V-FITC and propidium iodide. The distribution pattern of live and apoptotic cells was determined by FACS analysis. The same analyses was performed in HepG2 cells treated with $4 \mu \mathrm{M}$ of sorafenib and caspase inhibitors for $72 \mathrm{~h}$.Viable cells display no annexin and propidium iodide staining (Q3); early-stage apoptotic cells display high annexin and low propidium iodide staining (Q1); late-stage apoptotic cells display high annexin and high propidium iodide staining (Q2); DNA fragmentation is represented by high propidium iodide and low annexin staining (Q4). X-axis represents propidium staining (PE) and y-axis represents FITC staining. Data are representative of at least three independent experiments. GL2: negative control shRNA; shN3: Notch3 shRNA; C8: caspase 8; C9: caspase 9; NC: negative control (Z-FA-FMK). 
On this regard we further found that the pharmacological or molecular inhibition of GSK3 $\beta$, by lithium treatment or siRNA transfection respectively, increased sorafenib-induced apoptosis in both HCC cell lines (Fig.3C, E-F). Therefore, GSK3 $\beta$ activity seems to protect from sorafenib toxicity and the high level of the


Figure 2: In vivo evidence of the role of Notch3 in sorafenib resistance. (A-B) HepG2 and Huh7 cells were subcutaneously injected in the flank of NOD/SCID mice. (A) A difference in tumor growth was evident between GL2 negative control vs shN3 in both the analyzed cell lines. $\mathrm{P}=0.04$ and $\mathrm{P}=0.01$ at t-test for Huh7 and HepG2 respectively after 21 days of Sorafenib treatment. The results are representative data of two independent experiments (+/- S.E). (B) Quantification of growt fraction measured by Ki67 staining. (C) Quantification of tumor angiogenesis measured by immunohistochemical staining of MVD. Proliferation and angiogenesis were quantified as described in the methods. The numbers were the average of counting 7 field in each sample. *, $\mathrm{P}<0.05$ (by two tailed student's t test). (D) Immunohistochemistry of CD31 in representative cases of HepG2 and Huh7 xenografts. Scale bars= $20 \mu \mathrm{m}$. (E) Western blot analysis of two representative GL2 xenografts (1-2) and two representative shN3 xenografts (1-2). VEGFR2 was down-regulated in the shN3 xenografts compared to GL2 xenograts. GL2: negative control shRNA; shN3: Notch3 shRNA. 
inactive pGSK $3^{\text {Ser9 }}$ form found in Notch $3 \mathrm{KD}$ cells could play a critical role in the sorafenib increased lethality in these cells.

Finally, we examined whether sorafenib treatment modifies the expression of two proteins regulated by
Notch3, p21 and p53, known to affect the responsiveness to chemotherapeutics drugs [7, 14]. Supplemental Figure $2 \mathrm{~A}$ shows that the levels of p21 and p53 proteins were reduced in response to sorafenib exposure between 24 and 48 hours. On the mRNA side, semi-quantitative RT-PCR
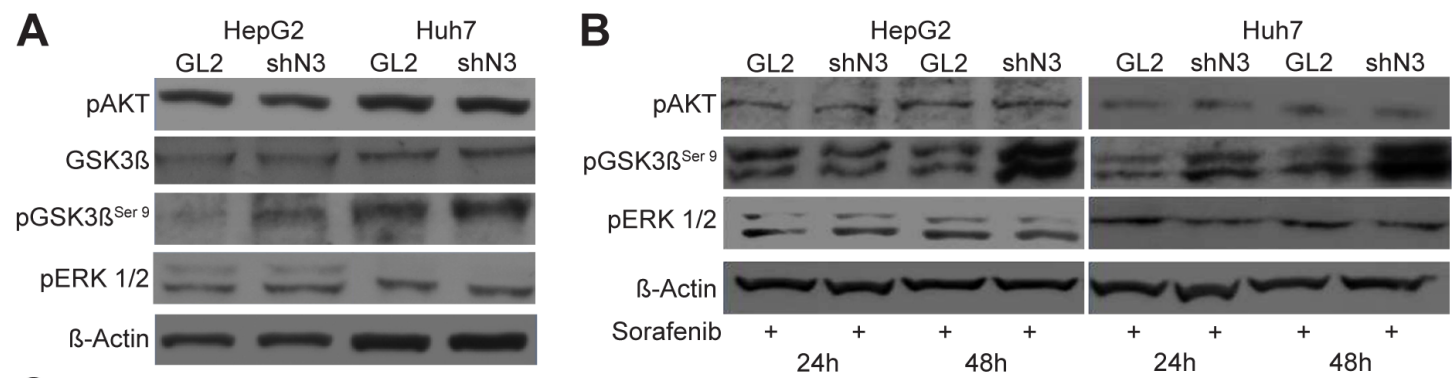

C
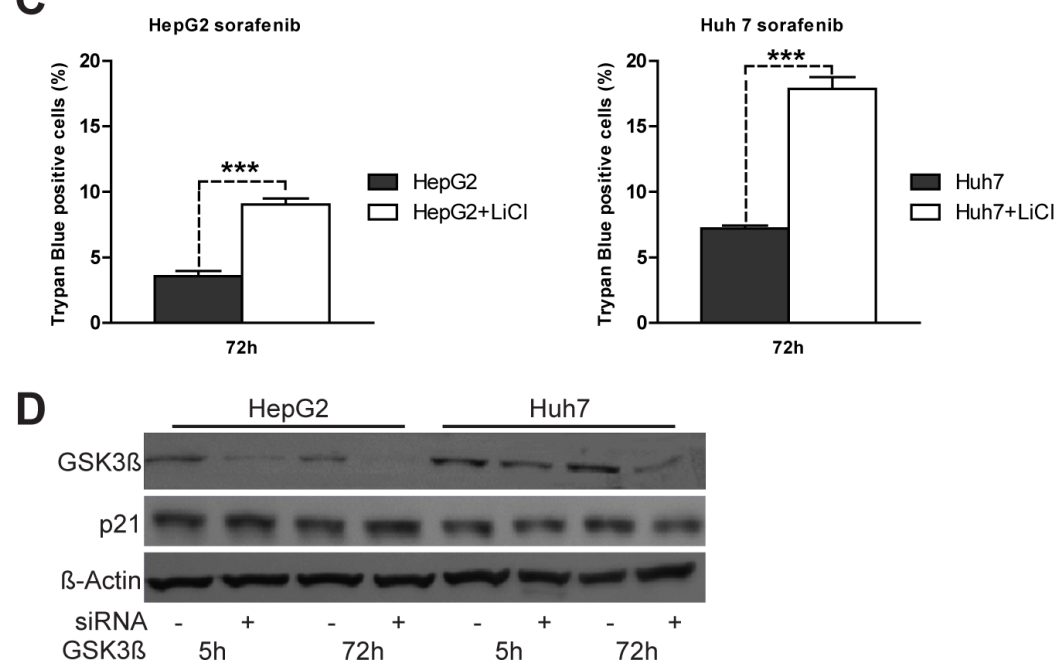

E
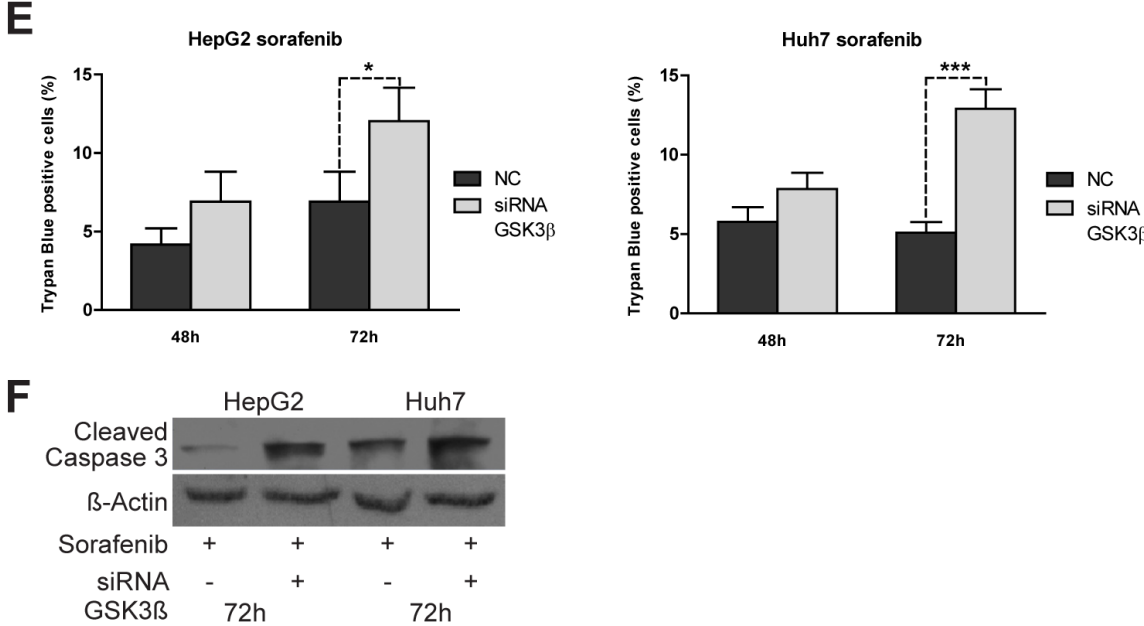

Figure 3: pGSK3ßser9 expression is regulated by Notch3 and enhances the effect of sorafenib. (A) pAkt, pERK, totalGSK $3 \beta$ and pGSK $3 \beta^{\text {ser9 }}$ protein levels in control and Notch3 KD cells were analyzed by western blotting. (B) The same proteins analyzed in (A) were evaluated after exposure to sorafenib for 24 and 48 h. GL2: negative control shRNA; shN3; Notch3 shRNA. (C) HepG2 and Huh 7 cells were treated with $20 \mathrm{mM}$ of LiCl. Cells were treated $5 \mathrm{~h}$ post lithium chloride treatment with $4 \mu \mathrm{M}$ of sorafenib for $72 \mathrm{~h}$ and cell death was assessed by trypan blue uptake. Results are shown as the means of three independent experiments (+/- S.E.). P values (by two tailed student's t test) were $\mathrm{p}<0.001$ for HepG2 and Huh7 VS LiCl treated cells. (D) HepG2 and Huh7 cells were transfected with GSK3 $\beta$ siRNA or scrambled RNA and GK3 $\beta$ knockdown and $\mathrm{p} 21$ protein levels were evaluated $5 \mathrm{~h}$ and $72 \mathrm{~h}$ post-transfection by western blot. (E) Cell viability by trypan blue uptake was scored three times as a function of $4 \mu \mathrm{M}$ of sorafenib treatment for the indicated time. Sorafenib treatment was started five hours post siRNA transfections. Results are shown as the means of three independent experiments (+/- S.E.). **, $\mathrm{P}<0.01$ (by two tailed student's t test). (F) Cells transfected with GK3 $\beta$ siRNA or scrambled RNA were treated with $4 \mu \mathrm{M}$ of Sorafenib for $72 \mathrm{~h}$ and the levels of cleaved caspase 3 were analyzed by western blotting. 
analyses in sorafenib treated cells revealed a decreased p21 mRNA expression but an increased expression of p53 mRNA (Suppl. Fig.2B). Interestingly, Notch3 depleted cells retain lower levels of p21 and the same levels of p53 then GL2 cells after exposure to sorafenib (Suppl. Fig.2C).

\section{P21 down-regulation contributes to sorafenib- induced cell death in HCC cells.}

To determine if lower p21 levels were functionally associated with the enhanced sorafenib sensitivity shown by Notch3 KD cells, we ablated endogenous p21 expression by transient siRNA transfection (Fig.4A). Transfected cells were treated with $4 \mathrm{uM}$ sorafenib for 48 $\mathrm{h}$ and $72 \mathrm{~h}$. p21 knockdown increased apoptosis induced by sorafenib as revealed by trypan blue uptake in three independent experiments and by increased levels of cleaved caspase 3 (Fig.4B-C). From the data it appears, that sorafenib treatment was more effective in Notch3 KD cells than in p21 silenced cells, suggesting that p21 was not the only reason for the enhanced sorafenib sensitivity following Notch3 depletion. In addition to transcriptional regulation by both $\mathrm{p} 53$ and Hes 1 proteins, p21 functions can be regulated at post-translational level by GSK3 $\beta$ [15]. However, the lower levels of p21 observed in sorafenib treated HepG 2 and Huh7 were not dependent by GSK3 $\beta$. Indeed GSK3 $\beta$ silencing did not down-regulate p21 protein expression (Fig.3D) suggesting that the effect of sorafenib in p21 depleted cells was independent of GSK3 $\beta$.

\section{Notch3 enhanced apoptosis of sorafenib treated cells depends on p21 and pGSK3ßSer9.}

To determine whether $\mathrm{p} 21$ and pGSK $3 \beta^{\text {Ser9 }}$ are major factors associated with the enhanced sorafenib sensitivity in Notch3 KD cells, we simultaneously ablated endogenous levels of both p 21 and GSK $3 \beta$ by transient siRNA transfection of HepG2 cells (Fig.4D). Five hours post-transfection cells were treated with $4 \mu \mathrm{M}$ of sorafenib
A

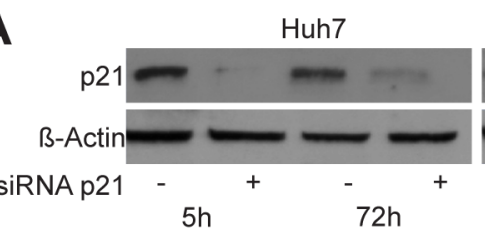

C

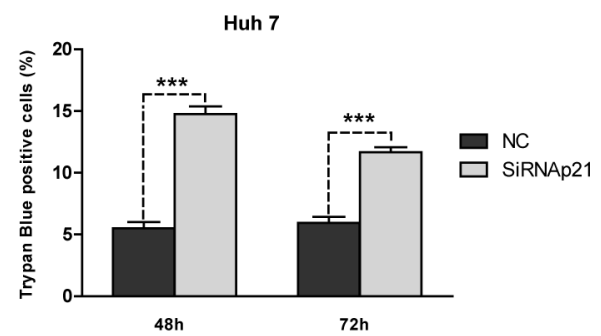

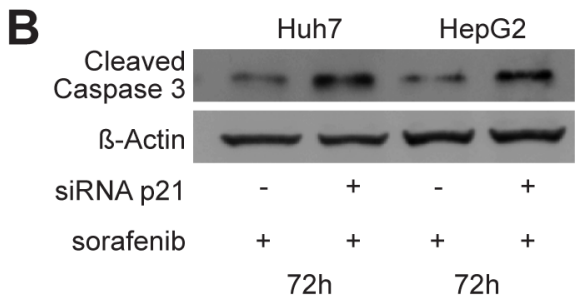

HepG2

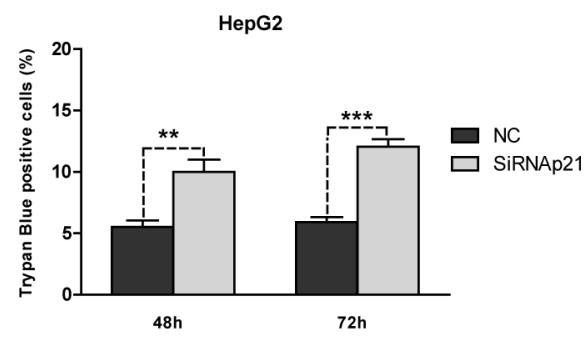

D

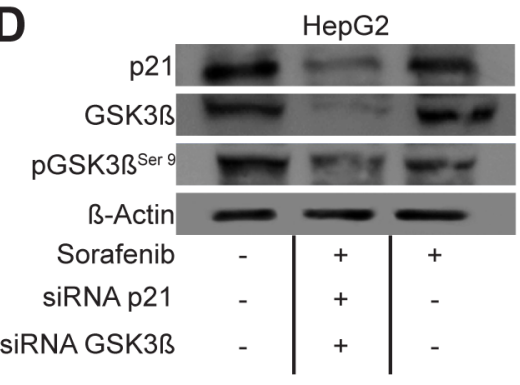

E



Figure 4: Effects of knockdown of p21 expression on apoptosis induced by sorafenib. (A) Huh7 and HepG2 were transiently transfected with a pool of siRNAs directed against p21 or negative control (NC) for $5 \mathrm{~h}$ and $72 \mathrm{~h}$. The level of p21 protein was evaluated by western blot.(B) Five hours post-transfection cells were treated with $4 \mu \mathrm{M}$ of Sorafenib for $72 \mathrm{~h}$ and the levels of cleaved caspase 3 were analyzed by western blotting. (C) The effect of p21 silencing on sorafenib $(4 \mu \mathrm{M})$ induced apoptosis were assessed by trypan blue uptake as the means of three independent experiments (+/- S.E.). **, $\mathrm{P}<0.01$ (by two tailed student's t test). (D) HepG2 cells were simultaneously transfected with p21 and GSK3 $\beta$ siRNA. $5 \mathrm{~h}$ post-transfection cells were treated with $4 \mu \mathrm{M}$ of Sorafenib for $72 \mathrm{~h}$ and the levels of p21, GSK3 $\beta$ and pGSK3 $\beta^{\text {ser9 }}$ were analyzed by western blotting. (E) Cell viability by trypan blue uptake was scored three times as a function of $4 \mu \mathrm{M}$ of sorafenib treatment for $72 \mathrm{~h}$. Results are shown as the means of three independent experiments (+/- S.E.). $* * *, \mathrm{P}<0.001$ (by two tailed student's $t$ test). 
for $72 \mathrm{~h}$. Down regulation of both $\mathrm{p} 21$ and GSK $3 \beta$ resulted in enhanced cell death as revealed by trypan blue uptake in three independent experiments (Fig.4E). Cell death upon sorafenib exposure increased 3.85 fold in cells silenced for both $\mathrm{p} 21$ and GSK $3 \beta$ compared to negative control (Fig.4E) $(\mathrm{p}<0.001)$. Considering that Notch3 depletion alone enhances sorafenib sensitivity 3.8 fold in HepG2 cells (Fig.1B), it seems that p21 and pGSK3 $\beta^{\text {Ser9 }}$ expression are the main reasons for the increased sorafenib sensitivity of Notch3 silenced cells in vitro.

\section{P53 is not required to enhance the cytotoxicity of sorafenib.}

To determine if lower p53 levels observed in sorafenib treated HepG2 and Huh7 cells were functionally associated with drug sensitivity, we ablated endogenous p53 expression by transient siRNA transfection (Suppl. Fig.3A). Transfected cells were treated with sorafenib for $48 \mathrm{~h}$ and $72 \mathrm{~h}$. Down-regulation of p53 reduced cellular death of both HepG2 and Huh7 only after 48 h of sorafenib
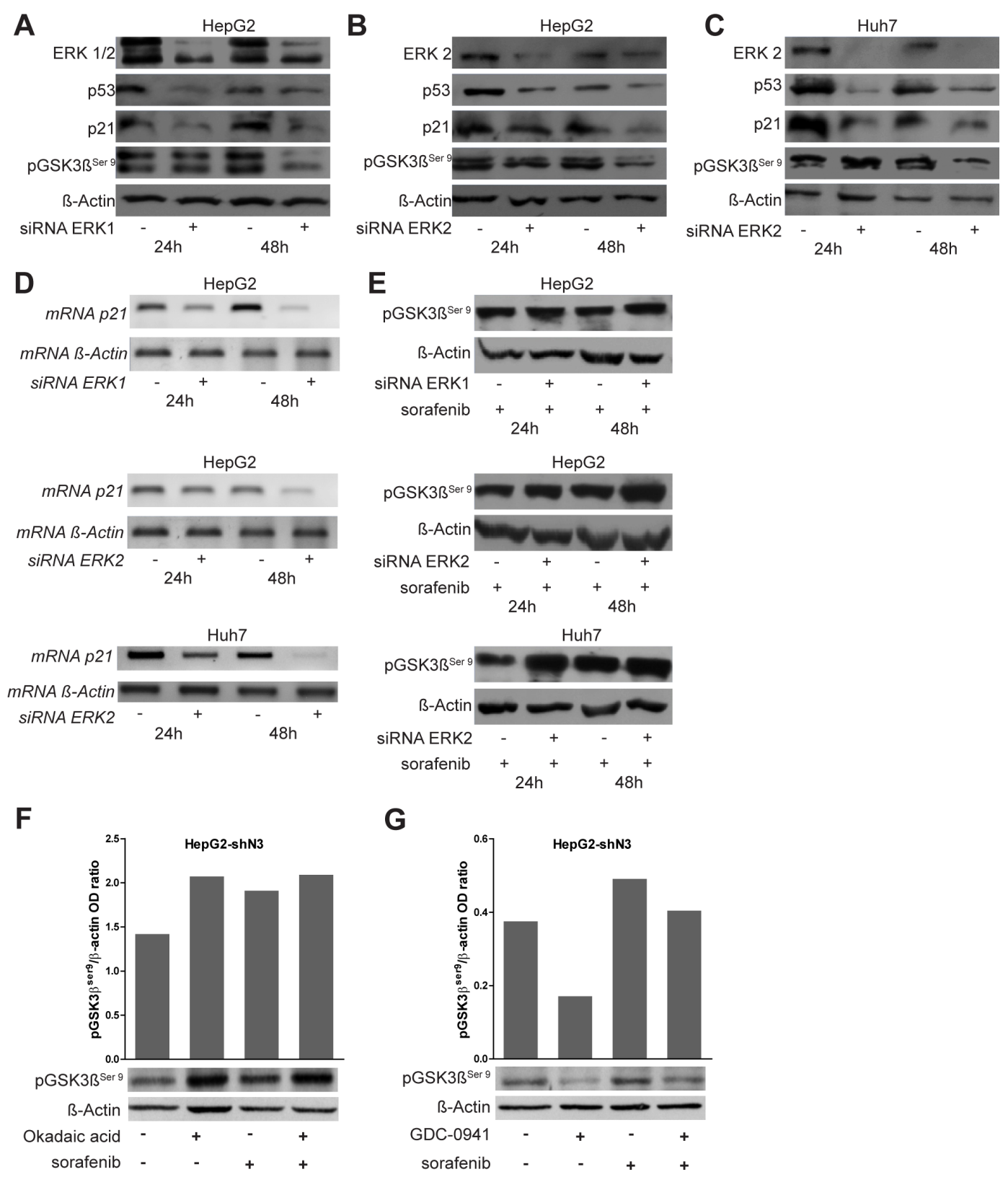

Figure 5: Role of ERK1/2 on p53, p21 and pGSK3ßser9 regulation. (A-B-C) HepG2 and Huh7 cells were transfected with a pool of ERK1 and ERK2 siRNAs or scrambled RNA and ERK1/2 knockdown, p21, p53 and pGSK3 $\beta^{\text {ser9 }}$ protein levels were evaluated $24 \mathrm{~h}$ and $48 \mathrm{~h}$ post-transfection by western blot. ERK1/2 silencing resulted in reduced levels of all the analyzed proteins. (D) Semi-quantitative RT-PCR expression analysis of p21 in ERK1/2 silenced cells. (E) pGSK3 $\beta^{\text {ser9 }}$ protein levels were analyzed after exposure to $4 \mu \mathrm{M}$ of sorafenib for 24 and $48 \mathrm{~h}$ in ERK1/2 silenced cells by western blotting. (F-G) pGSK3 $\beta^{\text {ser9 }}$ protein levels were evaluated $12 \mathrm{~h}$ post treatments with $60 \mathrm{nM}$ of Okadaic acid or $150 \mathrm{nM}$ of GDC-0941 or $4 \mu \mathrm{M}$ of sorafenib or combined treatments by western blot. The levels of proteins showed in panel $\mathrm{F}$ and $\mathrm{G}$ were quantified and expressed as a ratio with respect to $\beta$-actin control. 
exposure, as revealed by trypan blue uptake. After $72 \mathrm{~h}$ of treatment with sorafenib no difference were observed in cell death between control and p53 silenced cells (Suppl. Fig.3B) probably because sorafenib treatment downregulates p53 (Suppl. Fig.2B) causing the same effect of p53 silencing. Our findings are in line with a recent study showing that the sensitization effect of sorafenib on cisplatin can be mediated via both p53-dependent andindependent mechanisms [16]. These results strengthen the above observation indicating that $\mathrm{p} 21$ and $\mathrm{pGSK} 3 \beta^{\mathrm{Ser} 9}$ are the major effectors of the enhanced sorafenib sensitivity of Notch3 depleted HepG2 and Huh7 cells.

\section{Sorafenib regulates p21 and pGSK3ßSer9through ERK dependent and independent mechanisms.}

ERK proteins associates with and phosphorylates different proteins including GSK $3 \beta$ and p21 resulting respectively in their inactivation or degradation [17, 18]. In addition ERK1/2 act as activators of p53 and consequent cellular response [19]. To analyze if ERK1/2 are responsible for the alterations observed in the levels of p2 1 and pGSK3 $\beta^{\text {ser9 }}$ following exposure to sorafenib, their expression was assessed in ERK1/2 silenced cells.

ERK1/2 silencing resulted in decreased
pGSK3 $\beta^{\text {Ser9 }}$, p21 and p53 protein levels (Fig.5A-C). Semi-quantitative RT-PCR analyses revealed similar results for p21 gene transcription (Fig.5D). These results lets us to hypothesize that ERK1/ 2 mediate p21 downregulation via $\mathrm{p} 53$. However, sorafenib treatment of ERK $1 / 2$ silenced cells resulted in increased pGSK $3 \beta^{\text {Ser9 }}$ (Fig. 5E) suggesting that sorafenib regulates GSK3 $\beta$ phosphorylation through mechanisms independent of ERK1/2. Accordingly, pGSK $3 \beta^{\text {Ser9 }}$ was up-regulated in both untreated and sorafenib treated Notch3 KD cells although pERK1/2 protein levels were unchanged (Fig.3A-B). To further investigate the mechanism involved in GSK3 $\beta$ phosphorylation, HepG2 Notch3 silenced cells were treated with a phosphate inhibitor (Okadaic acid) ore with a kinase inhibitor (GDC-0941) alone or in combination with sorafenib. Phosphatase inhibition resulted in the same increase in pGSK $3 \beta^{\text {Ser9 }}$ observed in sorafenib treated cells. Interestingly combined treatment has the same effect on GSK $3 \beta$ phosphorylation than single compounds suggesting that this phenomenon is due to phosphatase inactivation by sorafenib (Fig. 5F). Moreover, in agreement with this observation, kinase inhibitor GDC0941, alone or in combination with sorafenib, reduces the levels of pGSK $3 \beta^{\text {Ser9 }}$ compared to sorafenib treated cells (Fig. 5G).
A

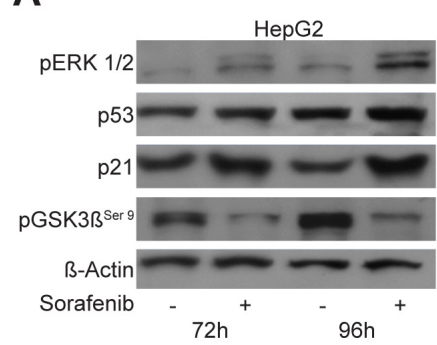

B

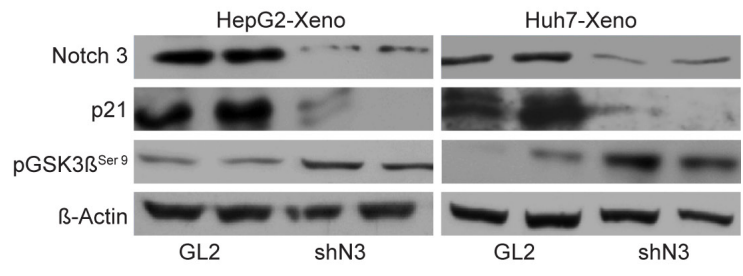

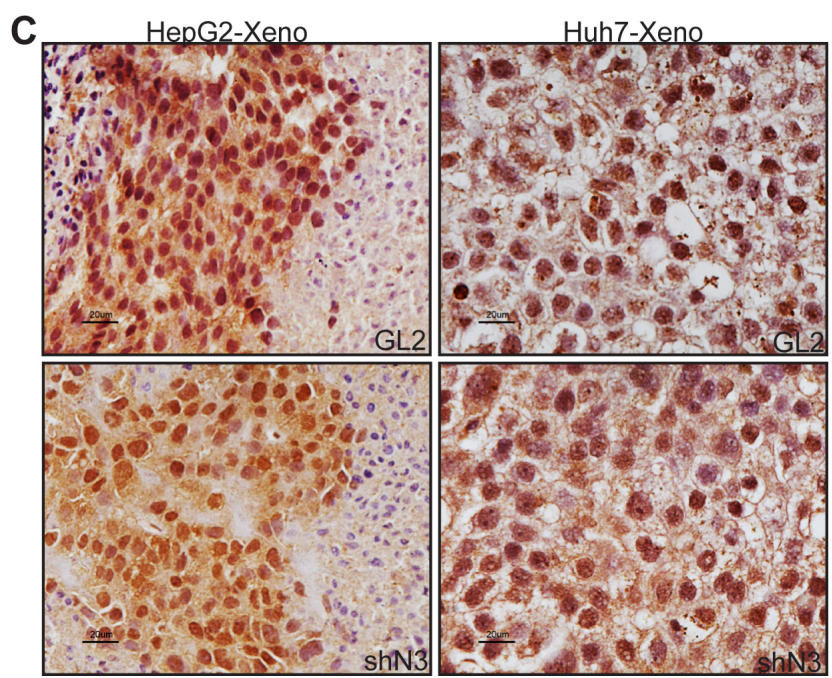

Figure 6: Sorafenib resistance evades blockade of ERK signaling. (A) pERK1/2, p53, p21 and pGSK3 $\beta^{\text {ser9 }}$ protein levels were analyzed after exposure to $4 \mu \mathrm{M}$ of sorafenib for $72 \mathrm{~h}$ and $96 \mathrm{~h}$ in HepG2 cells by western blotting. (B) Western blot analysis of Notch3, p21 and pGSK3 $\beta^{\text {ser9 }}$, expression in HepG2 and Huh7 xenografts evaluated after 21 days from the beginning of the treatment with $60 \mathrm{mg} / \mathrm{kg}$ of sorafenib. (C) Immunohistochemistry of pERK1/2 in representative cases of HepG2 and Huh7 xenografts. pERK1/2 accumulation was evident in the nucleus and in the cytoplasm. Scale bars= $20 \mu \mathrm{m}$. GL2: negative control shRNA; shN3; Notch3 shRNA. 


\section{Liver tumor cells confere resistance to sorafenib through pERK1/2 and p21 up-regulation and pGSK3ßSer9 downregulation.}

It was recently shown that ERK phosphorylation levels in tumor tissues correlate with the time to progression of patients affected by HCC. Indeed, in resistant HCC patients ERK1/2 activity was highly increased after 21 days of treatment with sorafenib [20]. We then explored possible mechanisms of sorafenib resistance in the HepG2 cells because an enhanced sorafenib sensitivity due to Notch3 ablation was higher in Huh7 cells than in HepG2 cells in vitro (Fig.1B). We found that ERK phosphorylation decreased after 24-48 h of treatment with sorafenib (Suppl.Fig.2A) and gradually increased after long sorafenib exposure (Fig.6A). In line with these observations, increased levels of p21 and decreased levels of pGSK $3 \beta^{\text {Ser9 }}$ were also observed while p53 protein levels were almost unaffected (Fig.6A).

\section{In vivo results}

Because $\mathrm{p} 21$ and pGSK3 $\beta^{\text {Ser9 }}$ contribute to Sorafenib resistance in vitro and since p 21 levels and pGSK $3 \beta^{\text {Ser9 }}$ are down-regulated and up-regulated respectively in
Notch3 KD cells, we examined their expression in tumor xenografts after sorafenib treatment. We found that Notch3 KD tumors had significant lower levels of p21 and increased levels of pGSK3 $\beta^{\text {Ser9 }}$ than GL2 tumors (Fig.6C). In line with the above reported in vitro results, Notch3 depletion did not affect phosphorylation and localization of ERK1/2 which resulted almost identical in GL2 and Notch3 tumors. (Fig.6D). The correlation between in vivo and in vitro studies suggest that, by keeping lower levels of p21 and higher levels of pGSK3 $3{ }^{\text {Ser9 }}$, Notch3 depleted cells did not acquire resistance to sorafenib.

\section{Notch 3 regulates $p G S K 3 \beta S e r 9$ and $\mathrm{p} 21$ protein levels in human HCC.}

To assess to what extent our in-vitro findings are representative of what occurs in human $\mathrm{HCC}$, we analyzed the expression of Notch3, p2 1 and pGSK3 $3 \beta^{\text {Ser9 }}$ proteins in 20 surgically resected HCCs by immunohistochemistry. We found a significant inverse correlation between Notch3 and $p G S K 3 \beta^{\text {Ser9 }}$ proteins accumulation (Spearman $\rho=-0.666, p<0.01)$ and a significant direct correlation between Notch 3 and $\mathrm{p} 21$ proteins expression (Spearman $\rho=0.681, p<0.01$ ) (Fig.7) suggesting that Notch3 participates in the control of GSK3 $\beta$ phosphorylation and p21 expression in human hepatocellular carcinoma.

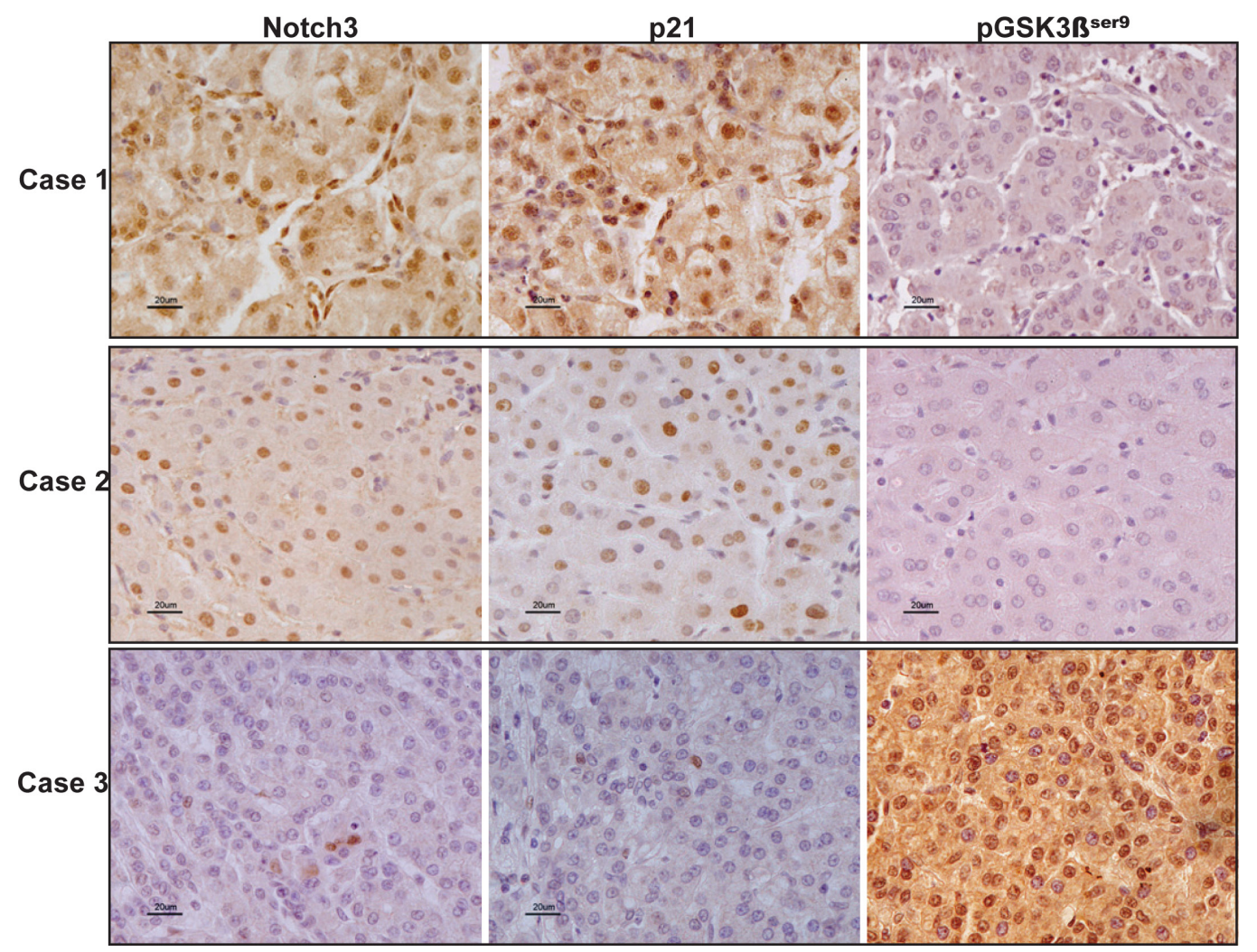

Figure 7: Expression profile of Notch3, p21 and pGSK3ßser9 in human HCC. Immunohistochemistry analysis in three representative cases showing Notch3, p21 and pGSK3 $\beta^{\text {ser9 }}$ expression in the same area. Scale bars $=20 \mu \mathrm{m}$. Case 1 and Case 2 show Notch 3 and p21 nuclear localization whereas they stain negative for pGSK3 $\beta^{\text {ser9 }}$. Case 3: HCC tissue with Notch3 and p21 negative hepatocytes results positive for $\mathrm{pGSK} 3 \beta^{\text {ser9 }}$ expression. 


\section{DISCUSSION}

It was recently shown, in large clinical trials, that sorafenib treatment significantly prolongs survival of patients with advanced HCC [1, 2], but the tumor response rate was short because of drug resistance [21]. Therefore, it is of great importance to either identify metabolic or signalling cellular pathways that could be targeted to enhance HCC sensitivity to sorafenib, or to understand the mechanisms of sorafenib resistance. Notch pathway is frequently deregulated in human cancer and inhibition of Notch signaling has been described as a promising strategy for tumors treatment [22]. It functions as an oncogene but can also act as a tumour suppressor. These apparently contradictory functions of Notch signalling strongly indicate that the effects of Notch activation are dependent on the cellular context [23]. In the present study we report that Notch3 depletion enhances sorafenib toxicity towards HCC cells both in vitro and in vivo. We found that either sorafenib treatment or Notch3 depletion resulted in p21 down-regulation and pGSK $3 \beta^{\text {Ser9 }}$ upregulation whereas sorafenib, but not Notch3 depletion, decreased p53 expression. Extensive studies have demonstrated that sorafenib mediates apoptosis through MEK/ERK-dependent and independent mechanisms. Examples include Gadd $45 \beta$, the translation initiator elF4E and Mcl-1 [24-26]. In line with this we showed that the reduced levels of p21 observed in sorafenib treated cells are ERK1/2 dependent. Conversely, sorafenib can increase pGSK3 $\beta^{\text {Ser9 }}$ levels whereas ERK1/2 silencing cannot. We then demonstrated that $\mathrm{p} 21$ silencing in association with GSK3 $\beta$ silencing or inactivation enhanced sorafenib sensitivity of HepG2 and Huh7 cell lines. Thus our observations are consistent with the active role assigned to p21 in inhibiting apoptosis $[20,27,28]$ and with the critical role played by GSK3 $\beta$ in cell survival [29]. As far as p53 is concerned, we found that sorafenib treatment in $\mathrm{HCC}$ cells resulted in enhanced mRNA expression but reduced protein levels. The induction of p53 mRNA is probably related to the pro-apoptotic stimulus induced by sorafenib. However, the lower level of p53 protein in sorafenib treated cells were consistent with reduced expression of the sorafenib targets ERK1/2. In support of the latter possibility, siRNA mediated ERK $1 / 2$ knockdown also reduced p53 expression in HepG2 and Huh7 cells. Moreover p53 depletion does not affect the sensitivity to long sorafenib exposure in $\mathrm{HCC}$ cells suggesting that p53 is not a key factor in mediating sorafenib response. Of interest here is our previous finding that Notch3 protects HCC from doxorubicin-induced death by controlling p53 levels [7]. Therefore, it seems that different pathways might be involved when Notch3 depletion is associated to the administration of a chemotherapeutic agents, like doxorubicin, or to the administration of a biologically active agent [29].

Overall, the above data indicate that the enhanced sorafenib sensitivity observed in Notch3 depleted HepG2 cells was mostly dependent on p21 and pGSK3.

Considering that resistance to sorafenib has been observed in different clinical trials we analyze whether p 21 and pGSK $3 \beta^{\text {Ser9 }}$ were involved in mediating resistance to the drug. ERK1/2 phosphorylation was rapidly reduced after exposure to sorafenib but progressively returned to baseline levels restoring the expression of p21, p53 and pGSK $3 \beta^{\text {Ser9 }}$ proteins in HepG2 cells. In contrast with this in vitro observation, Notch3 KD xenografts expressed lower levels of p21 and higher levels of pGSK $3 \beta^{\text {Ser9 }}$ than GL2 xenografts upon exposure to 21 days of sorafenib whereas no difference were observed in p53 expression between the two xenografts. In vivo, the association of sorafenib and Notch3 depletion resulted in slower tumor growth as demonstrated by reduced tumor volume and decreased Ki-67 labeled growth fraction

Inhibition of tumor angiogenesis is considered a major mechanisms of HCC response to sorafenib. HCC is a hypervascular tumor, and the use of anti-angiogenic therapy has been extensively studied [30]. However antiangiogenic therapy elicits forms of adaptive resistance that take to treatment failure [31]. Notch signaling participates in multiple aspects of vascular development including angiogenesis, differentiation and remodelling of vascular smooth muscle cells (VSMC) [32]. We proved that combination of sorafenib and Notch3 depletion significantly decreased both CD31 staining and VEGFR2 in vivo suggesting that Notch3 signaling by cancer cells plays a role in neo-angiogenesis.

The results presented in this study demonstrate, for the first time, that Notch3 inhibition enhances the effect of sorafenib in human HCC. Importantly, we present evidence that the effects of Notch 3 depletion in sorafenib response are mediated by $\mathrm{p} 21$ and $\mathrm{pGSK} 3 \beta^{\mathrm{Ser} 9}$ and, probably, by neo-angiogenesis inhibition. Our data suggest also that the synergistic activity of sorafenib with Notch3 mainly ends up in avoiding drug resistance. Altough $\gamma$-secretase inhibitors are today evaluated as promising inhibitors of Notch signaling in neoplastic disease [33,34], targeting Notch3 should be preferred for HCC therapy to avoid additional damage to non neoplastic cirrhotic liver [3].

\section{MATERIAL AND METHODS}

\section{Ethics Statement}

Investigation has been conduced in accordance with the ethical standards and according to the Declaration of Helsenki and according to national and international guidelines and has been approved by the authors institutional reviewed board. 


\section{Cell lines and Notch3 knockdown by retroviral transduction of shRNAs}

The human hepatocarcinoma cell lines HepG2 and Huh7 were obtained from American Type Culture Collection (ATCC, Rockville, MD, USA) at the end of 2011. Cells have not been authenticated by the authors. HepG2 cells were maintained in Eagle's Minimum Essential Media (MEM) while Huh7 cells were maintained in RPMI. Media were supplemented with $10 \%$ of fetal bovine serum (FBS), $100 \mathrm{U} / \mathrm{ml}$ of penicillin, and 100 $\mathrm{mg} / \mathrm{ml}$ of streptomycin (all reagent from ATCC) at 37 ${ }^{\circ} \mathrm{C}$ in $5 \% \mathrm{CO}_{2}$ incubator. Notch3 knock down (KD) was obtained using short hairpin oligonucleotides targeted to different Notch3 exons inserted into the pSuper. puro expression vector (OligoEngine, Seattle, WA) as previously described (7). Since two Notch3 specific shRNAs were equally effective in our previous study (7) we performed the experiments by selecting a single shRNA (shN3). Cells harbouring a pSuper.puro provirus expressing a GL2 luciferase specific shRNA were used as negative control (35).

\section{Compounds and cell death assays}

Sorafenib was obtained by Bayer Healthcare (BAY 43-9006, Italy), Okadaic acid was purchased from Sigma and GDC-0941 was obtained by Selleckem (Houston, TX, USA). Caspase 8 inhibithor (Z-IETD-FMK), Caspase 9 inhibitor (Z-LEHD-FMK) and negative control (Z-FAFMK) were from BD (Oxford, England).

Stably infected cell populations of HepG2 and Huh7 were seeded into 6-well dishes and allowed to attach for 24 hours before treatments. Cell death was revealed and quantified by multiple criteria. Our primary quantitative assay was trypan blue uptake. Apoptosis was also revealed by Annexin V-FITC (Bender Medsystems, Vienna, Austria) staining via FACS. Initiation of cell death was assessed by the appearance of cleaved caspase 8, 9 and 3 in western blots (36). Cellular necrosis was assessed by LDH (lactate dehydrogenase) release by control vs. sorafenib treated cells.

\section{Xenografts mouse model and treatments.}

Seven-to eight week-old NOD/SCID female mice (Harlan, Udine, Italy) were inoculated subcutaneously with $5 \times 10^{6} \mathrm{HepG} 2$ cells (GL2 or shN3) or with $3 \times 10^{6}$ Huh7 cells (GL2 or shN3). Treatment with sorafenib was initiated when tumor nodules reached $130 \mathrm{~mm}^{3}$ to $200 \mathrm{~mm}^{3}$ in volume as determined by ultrasonography (US) imaging. Sorafenib was given by oral gavages for 21 consecutive days at $60 \mathrm{mg} / \mathrm{kg}$ dose; tumor volume during treatment period was monitored weekly by using US imaging. At the end of the treatment, animals were sacrificed and each tumor mass was collected for laboratory analysis.

\section{Small interfering RNA transfections}

HepG2 and Huh7 cells were seeded into 6 well plates and transfected with $20 \mathrm{nM}$ of p53 (Invitrogen), p21(IDT, Thief River Falls , MN, USA), GSK3, ERK1/2 (Santa Cruz Biotechnology) siRNAs or scrambled siRNA (scRNA) using Lipofectamine2000 (InVitogen). Transfection efficiencies were greater than $90 \%$ as determined by co-transfection with a fluorescein-labelled siRNA (InVitrogen). Five hours were allowed to elapse before treatment with sorafenib $4 \mu \mathrm{M}$. Cells were collected at $5 \mathrm{~h}$ and $72 \mathrm{~h}$ post-transfection and proteins expression was analyzed by western-blot.

\section{SDS-PAGE and Western blot analysis}

Protein extraction and quantification were performed as previously described [7]. Primary antibodies were as follows: anti-Notch3 polyclonal antibody (sc-5593, Santa Cruz Biotechnology), anti-p21 monoclonal antibody (Clone SX118, Dako, Denmark), anti-p53 monoclonal antibody (Clone DO-7, Dako), anti-Cleaved Caspase 3 monoclonal antibody (9664, Cell Signaling Technology, Beverly, MA), anti-p-ERK monoclonal antibody (sc7383, Santa Cruz Biotechnology), anti-ERK1/2 polyclonal antibody (9102, Cell Signaling) anti-GSK3 $\beta$ monoclonal antibody (sc-7291, Santa Cruz Biotechnology), anti-p GSK3 $\beta$ monoclonal antibody (9323, Cell Signaling) anti-pAkt monoclonal antibody (2965, Cell Signaling), anti-VEGF Receptor 2 monoclonal antibody (2479, Cell Signaling), anti-CD31 polyclonal antibody (ab28364 Abcam, Cambridge, UK) and anti- $\beta$-actin monoclonal antibody (Clone AC-40, Sigma) Immunoreactivites were revealed with the EnVision dextran polymer visualization system (Dako).

\section{RNA analysis}

Total cellular RNAs were prepared with Trizol (InVitrogen, Paisley, Scotland) according to the manufacturer's instructions. Two micrograms of total RNA were reverse-transcribed using Superscript II (InVitrogen). Relative gene expressions were determined by semi-quantitative end-point PCR. PCR primers were as follow:

NOXA (FW 5'cgagaattcgagatgcctgggaag-3',

REV 5' cttggtaccggttcetgagcag-3')

bax (FW 5'acagggtttcaccaggatc-3',

REV 5'- gctgccacccgcaagaagac-3'), p21 (FW 5' aagaccatgtggacctgtca, 
REV 5' ggcttcctcttggagaagat),

p53 (FW 5' gacccaggtccagatgaagct,

REV 5' accgtagctgccctggtaggt),

$\beta$ ACTIN (FW 5'- gaggcactcttccagccttc-3',

REV 5'- ggatgtccacgtcacacttc-3').

\section{Patient samples}

Twenty patients of both sexes undergoing partial hepatectomy for HCC entered the study. Informed consent was obtained from each patient according to Italian guidelines and the latest version of the Helsinki Declaration. Exclusion criteria were a previous history of local or systemic treatments for HCC. Tissues sample were fixed in $10 \%$ formalin and paraffin-embedded for histopathology and immunohistochemistry.

\section{Immunohistochemistry (IHC)}

The presence and localization of pERK1/2, CD31and Ki67 (Dako) in tumor xenografts and the expression of Notch3, p21, pGSK3 $\beta^{\text {Ser9 }}$, in human HCCs, were immunohistochemically assessed on formalin-fixed, paraffin-embedded sections. Serial $4 \mu \mathrm{m}$ thick sections were processed for haematoxylin and eosin staining and for immunohistochemistry. Endogenous peroxidases were inhibited by incubating slides in $3 \% \mathrm{H}_{2} \mathrm{O}_{2}$-methanol for $20 \mathrm{~min}$ at $4^{\circ} \mathrm{C}$. For antigen retrieval, slides were immersed in $\mathrm{pH} 6.0$ citrate buffer $(\mathrm{pH} 6.0)$ and boiled using a microwave owen. Negative controls were obtained by omitting the primary antibody. Immunoreactivity was revealed with the EnVision system (DAKO), and diaminobenzidine (DAB) as chromogen (Sigma, ST Louis, USA).

Slides were counterstained in Meyer's hematoxylin, coverslipped and examined by light microscopy. Microvessel density (MVD), calculated as the average of microvessel counts in seven randomly selected 40x fields using a motorized stage (Marzhauser, Wetzlar, Germany), was used to represent tumor angiogenesis activity. Ki67 staining was quantified by image cytometry using Image J software (NIH, Bethesda, USA) on at least 7 randomly selected consecutive fields at $40 \mathrm{x}$ and expressed as the percentage of positive nuclei over the total nuclei (Labeling index:LI). Staining was assessed by two independent observers (L. G., C. G.) who evaluated the percentage of positive hepatocytes in each fields. Results represent the average of the percentage from 10 consecutive $20 \mathrm{x}$ magnification fields.

\section{Statistical analysis}

Differences between groups were analyzed using a double-sided Student t-test. Experimental data are expressed as the mean $\pm \mathrm{SE}$ from three independent experiments. T-test was also used to explore significant difference in tumor growth between control and Notch3 silenced xenografts. Spearman's correlation was used to explore the relationships between Notch 3 and p21 or Notch 3 and pGSK $3 \beta^{\text {ser }}$ expression in HCC tissues. $\mathrm{P}$-values less than 0.05 were considered statistically significant. Statistical analyses were performed using SPSS version 19.0.

\section{ACKNOWLEDGMENT:}

We thank Dott.ssa Marta Granata for technical assistance with western blots. This study was supported by a grant from Bayer- Healthcare Italy. Professor Luigi Bolondi has received research supports and fees for conference from Bayer, Bristol Myers Squibb, Roche, Bracco, Esaote, Syrtex and has consultance agreements with the same companies.

\section{REFERENCES}

1. Llovet JM, Ricci S, Mazzaferro V, Hilgard P, Gane E, Blanc JF, de Oliveira AC, Santoro A, Raoul JL, Forner A, Schwartz M, Porta C, Zeuzem S, Bolondi L, Greten TF, Galle PR, Seitz JF, Borbath I, Haussinger D, Giannaris T, Shan M, Moscovici M, Voliotis D, and Bruix J. Sorafenib in advanced hepatocellular carcinoma. N Engl J Med. 2008; 359(4):378-390.

2. Cheng AL, Kang YK, Chen Z, Tsao CJ, Qin S, Kim JS, Luo R, Feng J, Ye S, Yang TS, Xu J, Sun Y, Liang H, Liu J, Wang J, Tak WY, Pan H, Burock K, Zou J, Voliotis D, and Guan Z. Efficacy and safety of sorafenib in patients in the Asia-Pacific region with advanced hepatocellular carcinoma: a phase III randomised, double-blind, placebocontrolled trial. Lancet Oncol. 2009; 10(1):25-34.

3. Gramantieri L, Giovannini C, Lanzi A, Chieco P, Ravaioli M, Venturi A, Grazi GL, and Bolondi L. Aberrant Notch3 and Notch4 expression in human hepatocellular carcinoma. Liver Int. 2007; 27(7): 997-1007.

4. Miele L. Notch signaling. Clin Cancer Res. 2006; 12(4): 1074-1079.

5. Weijzen S, Rizzo P, Braid M, Vaishnav R, Jonkheer SM, Zlobin A, Osborne BA, Gottipati S, Aster JC, Hahn WC, Rudolf M, Siziopikou K, Kast WM, and Miele L. Activation of Notch-1 signaling maintains the neoplastic phenotype in human Ras-transformed cells. Nat Med. 2002; 8(9):979986.

6. Weng AP, Nam Y, Wolfe MS, Pear WS, Griffin JD, Blacklow SC, and Aster JC. Growth suppression of pre-T acute lymphoblastic leukemia cells by inhibition of notch signaling. Mol Cell Biol.2003; 23(2): 655-664.

7. Giovannini C, Gramantieri L, Chieco P, Minguzzi M, Lago F, Pianetti S, Ramazzotti E, Marcu KB, and Bolondi L. Selective ablation of Notch3 in HCC enhances 
doxorubicin's death promoting effect by a p53 dependent mechanism. J Hepatol. 2009; 50(5): 969-979.

8. Park JT, Chen X, Trope CG, Davidson B, Shih Ie M, and Wang TL. Notch3 overexpression is related to the recurrence of ovarian cancer and confers resistance to carboplatin. Am J Pathol. 2010;177(3): 1087-1094.

9. Wilhelm SM, Adnane L, Newell P, Villanueva A, Llovet JM, and Lynch M. Preclinical overview of sorafenib, a multikinase inhibitor that targets both Raf and VEGF and PDGF receptor tyrosine kinase signaling. Mol Cancer Ther.2008; 7(19): 3129-3140.

10. Villanueva A and Llovet JM. Targeted therapies for hepatocellular carcinoma. Gastroenterology.2011; 140(5):1410-1426.

11. Wilhelm SM, Carter C, Tang L, Wilkie D, McNabola A, Rong H, Chen C, Zhang X, Vincent P, McHugh M, Cao Y, Shujath J, Gawlak S, Eveleigh D, Rowley B, Liu L, Adnane L, Lynch M, Auclair D, Taylor I, Gedrich R, Voznesensky A, Riedl B, Post LE, Bollag G, and Trail PA. BAY 43-9006 exhibits broad spectrum oral antitumor activity and targets the RAF/MEK/ERK pathway and receptor tyrosine kinases involved in tumor progression and angiogenesis. Cancer Res. 2004; 64(19): 7099-7109.

12. Huang J, Zhang X, Tang Q, Zhang F, Li Y, Feng Z, and Zhu J. Prognostic significance and potential therapeutic target of VEGFR2 in hepatocellular carcinoma. J Clin Pathol.2011; 64(4): 343-348.

13. Chai H, Luo AZ, Weerasinghe P, and Brown RE. Sorafenib downregulates ERK/Akt and STAT3 survival pathways and induces apoptosis in a human neuroblastoma cell line. Int J Clin Exp Pathol. 2010; 3(4): 408-415.

14. Nefedova Y, Cheng P, Alsina M, Dalton WS, and Gabrilovich DI. Involvement of Notch-1 signaling in bone marrow stroma-mediated de novo drug resistance of myeloma and other malignant lymphoid cell lines. Blood. 2004; 103(9): 3503-3510.

15. Lee JY, Yu SJ, Park YG, Kim J, and Sohn J. Glycogen synthase kinase 3beta phosphorylates p21WAF1/CIP1 for proteasomal degradation after UV irradiation. Mol Cell Biol. 2007; 27(8): 3187-3198.

16. Dudgeon C, Peng R, Wang P, Sebastiani A, Yu J and Zhang L. Inhibiting oncogenic signaling by sorafenib activates PUMA via GSK3beta and NF-kappaB to suppress tumor cell growth. Oncogene. 2012; 31(46):4848-58.

17. Ding Q, Xia W, Liu JC, Yang JY, Lee DF, Xia J, Bartholomeusz G, Li Y, Pan Y, Li Z, Bargou RC, Qin J, Lai CC, Tsai FJ, Tsai CH, and Hung MC. Erk associates with and primes GSK-3beta for its inactivation resulting in upregulation of beta-catenin. Mol Cell. 2005;19(2): 159170.

18. Hwang CY, Lee C and Kwon KS. Extracellular signalregulated kinase 2-dependent phosphorylation induces cytoplasmic localization and degradation of p21Cip1. Mol Cell Biol. 2009; 29(12): 3379-3389.
19. Wu GS. The functional interactions between the p53 and MAPK signaling pathways. Cancer Biol Ther. 2004; 3(2): 156-161.

20. Caraglia M, Giuberti G, Marra M, Addeo R, Montella L, Murolo M, Sperlongano P, Vincenzi B, Naviglio S, Prete SD, Abbruzzese A, and Stiuso P. Oxidative stress and ERK1/2 phosphorylation as predictors of outcome in hepatocellular carcinoma patients treated with sorafenib plus octreotide LAR. Cell Death Dis.2011; 28:2 e150.

21. Palmer DH. Sorafenib in advanced hepatocellular carcinoma. N Engl J Med. 2008; 359(23): 2498.

22. Sonoshita M, Aoki M, Fuwa H, Aoki K, Hosogi H, Sakai Y, Hashida H, Takabayashi A, Sasaki M, Robine S, Itoh K, Yoshioka K, Kakizaki F, Kitamura T, Oshima M and Taket MM Suppression of colon cancer metastasis by Aes through inhibition of Notch signaling. Cancer Cell. 2011; 19(1): 125-137.

23. Radtke F and Raj K. The role of Notch in tumorigenesis: oncogene or tumour suppressor? Nat Rev Cancer. 2003; 3(10): 756-767.

24. Ou DL, Shen YC, Yu SL, Chen KF, Yeh PY, Fan HH, Feng WC, Wang CT, Lin LI, Hsu C and Chen A. L. Induction of DNA damage-inducible gene GADD45beta contributes to sorafenib-induced apoptosis in hepatocellular carcinoma cells. Cancer Res. 2010; 70(22): 9309-9318.

25. Rahmani M, Davis EM, Bauer C, Dent P and Grant S. Apoptosis induced by the kinase inhibitor BAY 43-9006 in human leukemia cells involves down-regulation of Mcl1 through inhibition of translation. J Biol Chem. 2005; 280(42): 35217-35227.

26. Panka, DJ, Wang W, Atkins MB and Mier JW. The Raf inhibitor BAY 43-9006 (Sorafenib) induces caspaseindependent apoptosis in melanoma cells. Cancer Res. 2006; 66(3): 1611-1619.

27. Suzuki A, Tsutomi Y, Akahane K, Araki T and Miura M. Resistance to Fas-mediated apoptosis: activation of caspase 3 is regulated by cell cycle regulator $\mathrm{p} 21 \mathrm{WAF} 1$ and IAP gene family ILP. Oncogene. 1998;17(8): 931-939.

28. Garner E and Raj K. Protective mechanisms of p53-p21$\mathrm{pRb}$ proteins against DNA damage-induced cell death. Cell Cycle. 2008; 7(3): 277-282.

29. McGill G and Fisher DE. p53 and cancer therapy: a doubleedged sword. J Clin Invest. 1999; 104(3): 223-225.

30. Zhu AX, Duda DG, Sahani DV and Jain RK. HCC and angiogenesis: possible targets and future directions. Nat Rev Clin Oncol. 2011;8(5): 292-301.

31. Grepin R and Pages G. Molecular mechanisms of resistance to tumour anti-angiogenic strategies. J Oncol. 2010;2010: 835680.

32. Rehman $\mathrm{AO}$ and Wang $\mathrm{CY}$. Notch signaling in the regulation of tumor angiogenesis. Trends Cell Biol. 2006;16(42): 293-300.

33. Chen SM, Liu JP, Zhou JX, Chen C, Deng YQ, Wang Y and Tao ZZ. Suppression of the notch signaling pathway 
by gamma-secretase inhibitor GSI inhibits human nasopharyngeal carcinoma cell proliferation. Cancer Lett. 306(1): 76-84.

34. Kondratyev M, Kreso A, Hallett RM, Girgis-Gabardo A, Barcelon ME, Ilieva D, Ware C, Majumder PK and Hassell JA. Gamma-secretase inhibitors target tumor-initiating cells in a mouse model of ERBB2 breast cancer. Oncogene. 2012; 31(1):93-103.

35. Elbashir SM, Lendeckel $\mathrm{W}$ and Tuschl T. RNA interference is mediated by 21- and 22-nucleotide RNAs. Genes Dev. 2001; 15(2): 188-200.

36. Giovannini C, Lacchini M, Gramantieri L, Chieco P and Bolondi, L. Notch3 intracellular domain accumulates in HepG2 cell line. Anticancer Res. 2006; 26(3A): 2123-2127. 